\title{
Structured Product Labeling Potency Terminology
}

National Cancer Institute

\section{Source}

National Cancer Institute. Structured Product Labeling Potency Terminology. NCI

Thesaurus. Code C54458.

Terminology used for representation of the information on pharmaceutical product potency in the framework of the Structured Product Labeling documents. 